\title{
Tuberculosis: Common disease with uncommon presentation: A case series
}

\author{
Dr. Sukla Naskar ${ }^{1}$, Dr. Pasang L. Sherpa ${ }^{2}$, Dr. Soumya Kanti Kundu ${ }^{3}$, \\ Dr. Arunima Mukherjee ${ }^{4}$, Prof. Keya Basu ${ }^{5}$ \\ ${ }^{1}$ Assistant Professor, Department of Pathology, Calcutta National Medical College Kolkata, WB \\ ${ }^{2}$ Assistant Professor, Department of Medicine, North Bengal Medical College, WB \\ ${ }^{3}$ Consultant, Department of Nuclear Medicine, RTIICS, NH, Kolkata \\ ${ }^{4}$ Assistant Professor, Department of Surgery, Calcutta National Medical College Kolkata, WB, \\ ${ }^{5}$ Professor and Head, Department of Pathology, Calcutta National Medical College, Kolkata, WB.
}

\begin{abstract}
Tuberculosis is an important cause of morbidity in the Indian society particularly among the low socioeconomic population and among the immunocompromised. Unlike pulmonary tuberculosis, extrapulmonary tuberculosis is less common and difficult to diagnose. We report a case series of primary extrapulmonary tuberculosis affecting rare sites of the body where tuberculosis is usually not demonstrated. Tuberculosis should be always considered as an important suspect in the etiology and differential diagnosis of any organic lesion.
\end{abstract}

Key Words: Extra pulmonary Tuberculosis, Rare Sites, Developing country

\section{Introduction}

Tuberculosis remains a significant public health problem worldwide, especially in a developing country like India. Usually lungs are the most common organs involved, while the extrapulmonary involvement is secondary and spread occurs by hematogenous, lymphatic or contagious spread from underlying focus. However $15-20 \%$ of all cases of primary Tuberculosis are extrapulmonary, the most common sites being the lymph nodes, pleura, abdomen and central nervous system ${ }^{1}$. Earlier, certain tissues like heart, striated muscle, thyroid and pancreas were considered relatively immune to tuberculosis ${ }^{2}$. However it has now being demonstrated that tuberculosis can virtually involve any organ of the body and manifests itself in various forms.

We report 6 cases of extrapulmonary tuberculosis. The unique feature is that in all the cases, tuberculosis is primary and demonstrated at very rare sites. All the patients were found to be immunocompetent.

Case 1: Primary Tuberculosis of thyroid gland presenting as a solitary thyroid nodule.

1.1: History: A male patient aged 32 years presented with a swelling in front of the neck for 6 months. He did not give any history of local pain, fever, cough, night sweats or weight loss. Local examination revealed a non tender and mobile solitary nodule in right lobe of thyroid gland $3 \times 4 \mathrm{cms}$ in size. There were no clinical features suggestive of hypothyroidism or hyperthyroidism. All routine blood investigations including ESR were normal. Thyroid function test was normal. USG of thyroid gland revealed a solitary heterogeneous, hypoechoic nodule in right lobe of thyroid gland. FNAC from nodule followed by LG stain showed follicular epithelial cells with presence of granuloma, consisting of aggregates of epithelioid cells and multinucleated giant cells. Z-N stain showed multiple acid fast bacilli(AFB). Chest X-ray was normal; USG of whole abdomen did not reveal any lymph nodes or other evidence of tuberculosis. Thus, a diagnosis of primary tuberculosis of thyroid gland presenting as a solitary nodule was established. 
Photomicrograph showing thyroid nodule and granuloma with thyroid follicular cells in inset

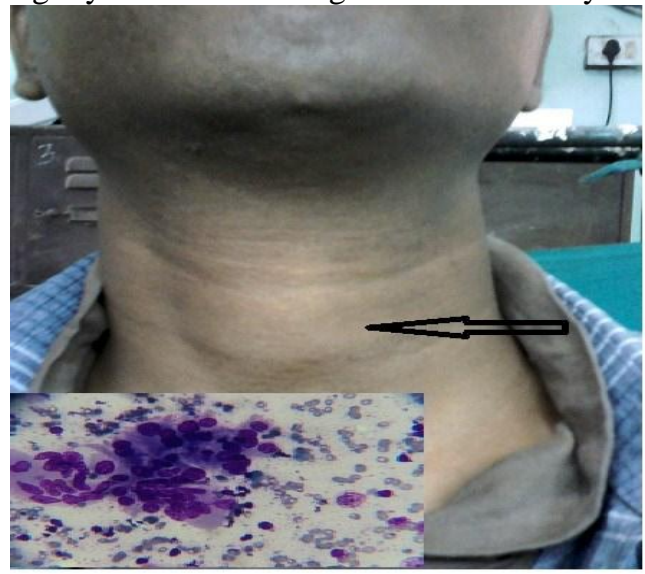

\section{2: Discussion:}

Although primary tuberculosis of thyroid gland is unusual, but it should be considered as a probability in any midline neck swelling especially in a developing country like India. The first case of tuberculosis of thyroid gland was described by Lebert in 1862 which was a manifestation as a part of disseminated tuberculosis. Clinical presentation is variable and usually consists of local symptoms related to enlargement of the gland, while thyroid function is rarely affected ${ }^{3}$. It can also present as a localized swelling mimicking thyroid carcinoma. Rarely presents as an acute abscess or as a thyroid nodule. In most of the reported cases patients were middle aged women with euthyroid status.

The differential diagnosis of tuberculosis of thyroid gland includes bacterial or fungal thyroiditis, subacute granulomatous thyroiditis. But the presence of caseous necrosis excludes those causes and simultaneous demonstration of acid fast bacilli confirms the diagnosis ${ }^{4}$.

Similar cases of primary thyroid tuberculosis without any evidence of tuberculosis in any other organ were reported by Aisaka $\mathrm{H}$ et al and Shahid $\mathrm{M}$ et al from Rawalpindi, Pakistan ${ }^{5}$.

Most of the reported cases of tuberculosis of thyroid gland were diagnosed histopathologically after thyroidectomy ${ }^{6}$. It is rare to establish such cases only by FNAC as it is difficult and seldom possible to find acid fast bacilli on aspiration ${ }^{2}$.

Pre operative diagnosis can avoid unnecessary surgery as it has been recognized that anti-tuberculous drugs alone can be efficacious and adequate ${ }^{6}$.

\section{CASE 2: Tuberculosis of skeletal muscle.}

Skeletal muscle is considered as an unfavourable site for survival and multiplication of Mycobacterial tuberculosis. About $3 \%$ of the patients with tuberculosis have musculoskeletal involvement mostly spondylitis, osteomyelitis, or arthritis ${ }^{7}$. Primary skeletal muscle tuberculosis is extremely rare and earlier studies have reported only four cases of muscle tuberculosis in 2224 autopsy specimens from tuberculosis patients ${ }^{7}$. Tuberculosis involvement of skeletal muscle is by direct extension from underlying bone, synovial lining of joints or tendon sheaths, by direct inoculation and rarely by hematogenous dissemination.

We report this case of tuberculosis of deltoid muscle in an immunocompetent patient.

\subsection{1: Tuberculous abscess of deltoid muscle}

2.1.2: History : A 32 year old female, previously healthy presented with two months history of gradually increasing painful swelling in right upper arm. She had a history of low grade fever for same duration. However she did not give any history of night sweats, weight loss, trauma or intramuscular injection at the local site. There was no history of contact with tuberculosis or any past history of pulmonary tuberculosis. Examination revealed a small tender cystic swelling in lateral aspect of right deltoid muscle $5 \times 4 \mathrm{cms}$ in size with erythema of overlying skin. Plain X-ray of shoulder joint and upper arm was normal. Routine blood tests showed mild neutrophilic leucocytosis with mean ESR of $60 \mathrm{~mm}$. HIV (I \& II) serology was negative. Other blood tests like sugar, urea, creatinine and LFT were normal. FNAC from the lesion lead to aspiration of pus. LG stain showed degenerated lymphocytes and epithelioid cells in necrotic background. Z-N stain showed plenty of acid fast bacilli.

Mantoux test was positive. Chest X-ray and USG of whole abdomen were done later which was completely normal. Thus a diagnosis of tuberculous abscess of right deltoid muscle in an immuno-compitent female was established. 


\section{Photomicrograph showing deltoid swelling and AFB in Z-N stain.}

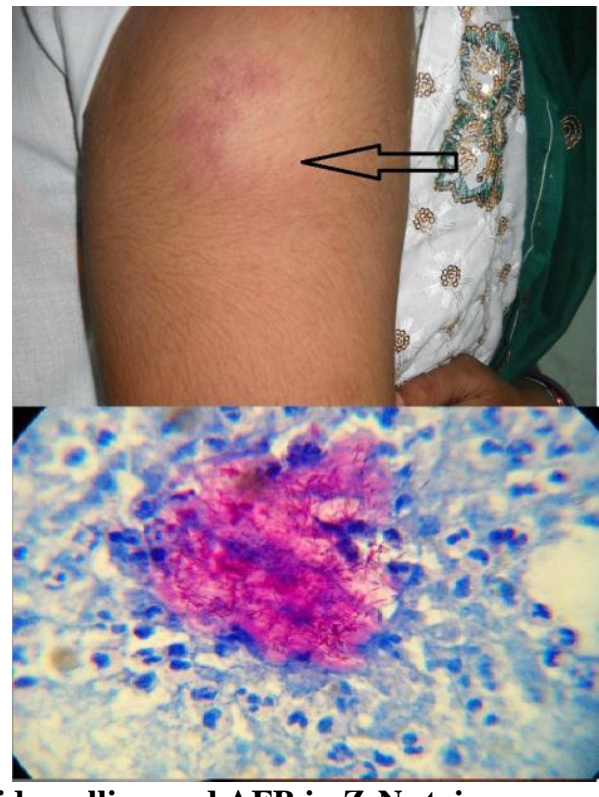

\subsection{1: Tuberculous myositis of thigh}

2.2.2: History:A 40year old male patient previously healthy, without prior history of contact with tuberculosis was admitted with tender poorly localized swelling in lateral aspect of right thigh for 2 months. He had a history of low grade fever for same duration. There was no history of weight loss, night sweats, local trauma or intramuscular injection. FNAC from the lesion followed by Z-N staining revealed acid fast bacilli and presence of granuloma in LG stain. Routine blood investigation was normal and HIV serology was negative. Chest X-ray and USG of whole abdomen were normal. X-rays of femur and pelvic joint were found to be normal.

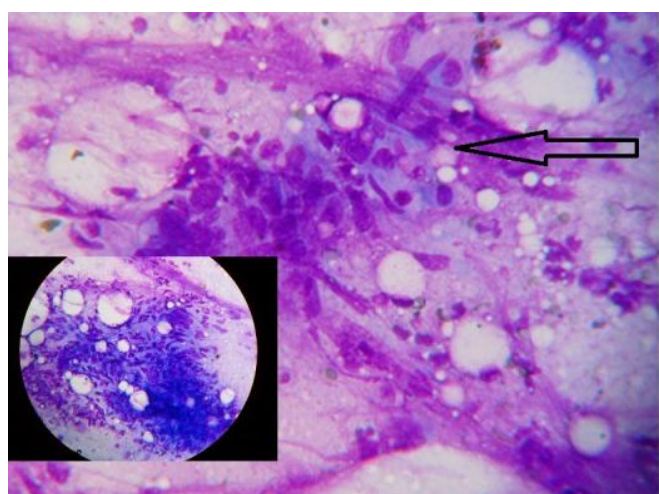

\section{Photomicrograph showing granuloma in LG stain in thigh swelling}

\subsection{Discussion:}

The selective involvement of skeletal muscle by tuberculosis without co existing active bony or extraskeletal tuberculosis is very rarely seen. Skeletal muscle was considered as an unfavourable site for survival and multiplication of tubercular bacilli. Few cases of primary tuberculosis of muscle may be transmitted by syringes by direct penetration of tubercle bacilli into the muscle tissue by hypodermic injections. Such a case was described by Coope after penicillin therapy ${ }^{8}$. Five cases of non respiratory tuberculosis with mycobacterial tuberculosis were reported after penetrating lesions of the skin. Three of the patients developed the disease after being treated repeatedly with intramuscular corticosteroid injections and two had cut themselves accidentally with a knife ${ }^{9}$.However few cases of isolated tuberculosis of muscle without any evidence of primary focus have been reported. By far, the commonest site of involvement is the thigh muscle ${ }^{10}$.

We report these cases as it is unusual to develop primary tuberculosis of deltoid muscle and quadriceps femoris( thigh) in absence any underlying primary focus of infection or of any precipitating factors like trauma at a local site. 


\section{Case 3: Tuberculous involvement of left occipital region}

3.1: History: A 22 year old female attended our clinical with a painful swelling in left occipital region which had gradually progressed over three months. There was no history of fever. weight loss, night sweats or local trauma. She did not give any history of contact with tuberculosis. Examination revealed a firm, tender swelling around $6 \times 7 \mathrm{cms}$, mobile, free from underlying structures and without any overlying skin changes. There was no regional lymphadenopathy. Routine blood investigation showed ESR of 50mm .HIV serology was negative. $\mathrm{X}$-ray of skull and plain CT scan of brain were normal. FNAC from the lesion was conducted which revealed acid fast bacilli in necrotic background in Z-N stain.

Similarly, we looked for evidence of underlying primary site of tuberculosis but chest X-ray and USG of whole abdomen were normal. Neither there was any involvement of other lymph nodes.

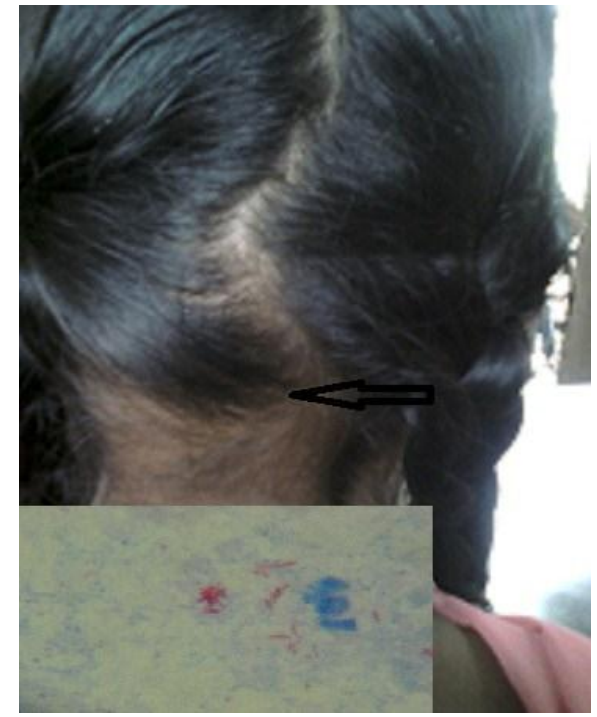

\section{Photomicrograph showing occipital swelling and AFB in Z-N stain}

\section{2: Discussion}

Soft tissue involvement in tuberculosis is generally associated with an underlying disorder, immunosuppressive therapy or local injury ${ }^{10}$. We report this rare case of tuberculous involvement of soft tissue in left occipital region as to our knowledge this is the rarest site of involvement and also the first case being reported so far.

\section{Case 4: Primary tuberculosis of gall bladder.}

Gall bladder is an unusual organ to develop primary tuberculosis. Gall bladder harbouring benign lesions are prone to develop tuberculosis. Such association is reported for gallstones ${ }^{11}$, diffuse papillomatosis of gall bladder ${ }^{12}$ and opisthorchiasis ${ }^{13}$.

We report a case of primary tuberculosis gall bladder which was coincidentally discovered during histopathological examination of a post cholecytectomized specimen of gall bladder.

4.1.: History : A 43 year old female was admitted for cholecystectomy .She had a history of recurrent right upper abdominal pain and vomiting. However there was no history of fever, anorexia and weight loss. Routine blood investigations were normal. USG of abdomen revealed features of calculus cholecystitis, there was no evidence of enlargement of intra abdominal lymph nodes. She was prepared for cholecystectomy after her acute symptoms were controlled and after cholecystectomy the specimen of gall bladder was sent for histopathological examination. HPE revealed granuloma with multinucleated Langan's type of giant cells in association of inflammatory cells.

On finding features of tuberculosis on HPE, she was investigated further to look for other underlying organs involved by tuberculosis. So X-ray of chest and repeat ultrasonography of abdomen were done which turned out to be normal. She was followed up for another year to look for reactivation of tuberculosis at any other site. However she remained asymptomatic.

Thus, a diagnosis of primary tuberculosis of gall bladder was established.

\section{2: Discussion}

The gall bladder may be infected by tuberculosis as a part of miliary tuberculosis, abdominal tuberculosis or through entero-hepatic route. Presentation may be asymptomatic with a histological diagnosis or 
may be symptomatic with usual systemic features of tuberculosis like low grade fever, malaise, anorexia. In most of the reported cases the commonest presentation has been that of cholecystitis and very few cases have presented as a gall bladder mass with carcinoma of gall bladder as a differential diagnosis. Cholelithiasis is a common association of both tuberculosis and malignancy of the gall bladder. The clinical presentation also mimics each other ${ }^{14}$.

Although tuberculosis of gall bladder is a rare entity and not adequately documented ${ }^{15}$, hence all resected gall bladder specimen should be subjected to histological examination in endemic areas like India to rule out tuberculosis.

\section{Case 5 : Isolated tuberculosis of ovary in a perimenopausal lady presenting as menorrhagia.}

Genitourinary tuberculosis is the second most frequent location for extrapulmonary tuberculosis after lymphatic system ${ }^{16}$. .Although genitourinary tuberculosis is common, isolated ovarian tuberculosis is rare.

We report this case of ovarian tuberculosis which was co incidentally discovered in a specimen of ovary performed after total abdominal hysterectomy with bilateral salpingo- oopherectomy (TAH \& BSO) in a perimenopausal woman.

5.1: History: A 45 year old lady, previously healthy was admitted with a history of menorrhagia for last six months. There was no history of pain abdomen,or any clinical features suggestive of thyroid dysfunction. Her routine blood investigation revealed mild anemia and her thyroid function test was normal. USG of abdomen showed mildly bulky uterus with normal endometrial thickness and normal ovaries, there was no evidence of tubo- ovarian mass. Endometrial biopsy was performed which was normal. There was no evidence of malignancy. Since her problem continued, she was then treated with three cycles of combined hormone therapy. Unfortunately she remained symptomatic and after 6 months she was prepared for TAH \& BSO. After surgery the specimen was sent for HPE, which showed granuloma with multinucleated Langhan's type of giant cells in ovarian stroma.

Thereafter search for primary focus of tuberculosis was done. Chest X-ray was normal, peritoneal biopsy was normal. No other focus of tuberculosis could be identified.

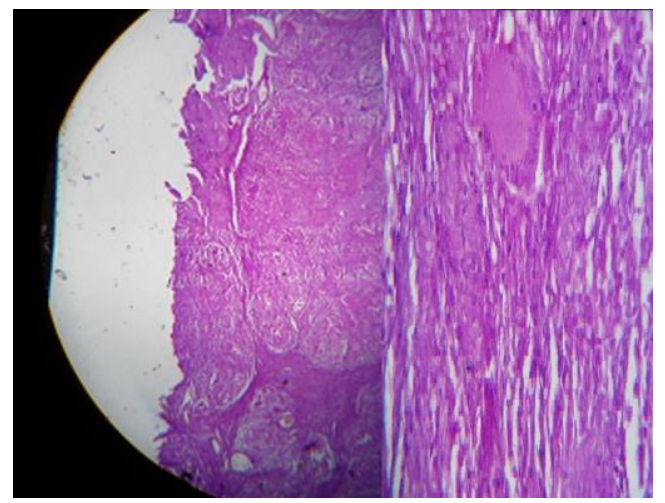

Photomicrograph showing giant cell and granuloma in ovary in H\&E stain

\section{2: Discussion:}

Involvement of ovaries by tuberculosis in majority of the cases is a part of genital or peritoneal tuberculosis, secondary to hematogenous, lymphagenous or contagious spread from fallopian tubes or peritoneum. The disease may remain asymptomatic or may lead to pelvic pain, fever, menstrual disturbances or vaginal discharge. Very few cases of isolated ovarian tuberculosis mimicking ovarian malignancy with raised CA125 level without any primary lung involvement have been reported ${ }^{17}$.

Our case is unique as it presented with menorrhagia which has not been reported so far.

\section{Case 6 : Tuberculous abscess of breast}

Tuberculosis of breast is an uncommon presentation of tuberculosis, even in the countries where the incidence of pulmonary and extrapulmonary tuberculosis is high ${ }^{18,19}$. The incidence of tuberculous mastitis is around $4 \%$ in tuberculosis endemic countries like India ${ }^{19}$.

Here, we report a case of primary tuberculous abscess of breast in young immunocompetent female patient, presenting as a nodular breast swelling.

6.1: History: A 27year old lady, previously healthy, attended our clinic with a breast nodule in lower outer quadrant of right breast for last two months. Initially it was painless, but within a period of two months it 
enlarged and became painful. She gave a history of occasional fever for same duration. There was no history of weight loss, night sweats or history of contact with tuberculosis.

Examination revealed a mobile, firm and tender breast lump around $5 \times 6 \mathrm{cms}$ in lower outer quadrant of right breast with erythema of overlying skin. There was no axillary lymphadenopathy. She was afebrile. Routine blood count revealed mild neutrophilic leucocytosis and ESR of $35 \mathrm{~mm}$. Her blood sugar was normal and HIV serology was non reactive. Chest X-ray was normal. USG of right breast showed a heterogenous hypoecheoic breast mass with some cystic component and edema of right pectoralis muscle. USG guided aspiration was done at the same setting which led to aspiration of pus like material which was sent for Gram stain, Z-N stain and culture sensitivity. Gram stain showed plenty of pus cells and Z-N stain revealed plenty of acid fast bacilli.

Thus, a diagnosis of primary tuberculous abscess of breast was established since no other foci of tuberculosis were found. She responded well to anti tubercular therapy and there was complete resolution of the abscess.

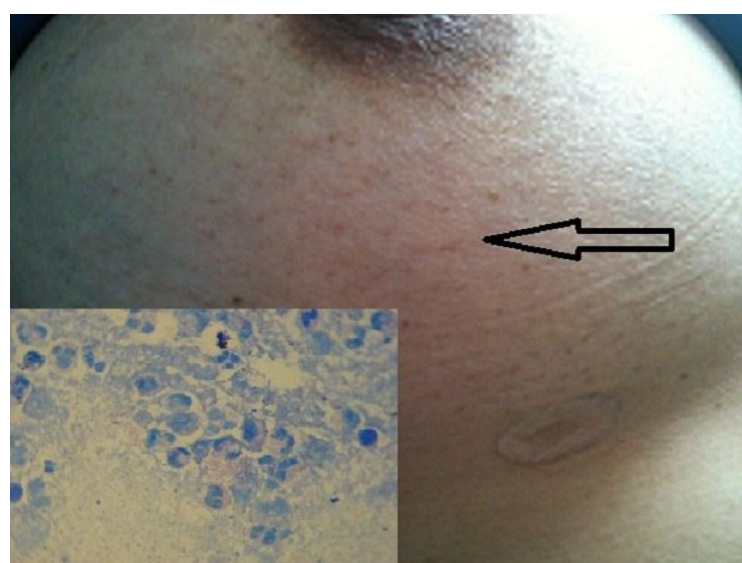

Photomicrograph showing breast swelling and AFB in Z-N stain

\section{2: Discussion}

Breast tuberculosis frequently occurs in women of reproductive age and is uncommon in prepubescent and elderly women. Breast tuberculosis can be categorized into three forms namely nodular, disseminated and abscess variety ${ }^{20}$. Clinical presentation ranges from a solitary, ill defined, unilateral hard lump, usually in upper outer quadrant to an irregular hard lump indistinguishable from carcinoma breast. It can also present as multiple lumps but bilateral involvement is uncommon.

\section{References}

[1]. Fanning A.Tuberculosis: 6 Extrapulmonary disease.CMAJ 1999;160: 1597-1603.(PMC free article)(PubMed)

[2]. Corton RS, Kumar V. Robbins S.L. In Robbins Pathological basis of the disease $5^{\text {th }}$ Edition 1994. W.B.Saunders company U.S.pp326 and 82 .

[3]. Orlandi F, Fiorini S, Gonzatto I, Saggiorato E, Pivano G, Angeli A, Pasquali R. Tubercular involvement of the thyroid gland: a report of two cases. Horm Res 1999; 52(6): 291-299

[4]. E Razmpa,H Sharifian,M Sadaghi, Hasanabadi, A Ilami and S.H. Shahinfar. Clinical and paraclinical aspects of thyroid tuberculosis. Acta Medica Iranica;2007: 45(5): 389-394

[5]. Shahid Mahmood, Syed Raiazul Hasan, Muhammad Sikandar Aslam. Primary tuberculosis of thyroid gland.Rawal Med J 2013;38: 84-85.

[6]. El Kohen A, Essakali L, Amarti A, Benchekroun L, Jazouli N, Kzadri M. Thyroid tuberculosis associated with microcarcinoma of the thyroid gland: a case report .Rev Laryngol otol Rhinol(Berd) 2001;122(3): 205-8.

[7]. Culotta A. La tuberculosis musculorae. Rev Pathol Tuberc;1929:3: 1-26

[8]. Coope, P.J.(1946) : Tuberculous abscess formation following Penicillin therapy. Proceedings of the Royal College of Society of Medicine( section of Paediatrics);40:161

[9]. De Jong J.W, van Altena R. Non respiratory tuberculosis with mycobacterium tuberculosis after penetrating lesions of the skin: five case histories. Int J Tuberc Lung Dis 2000 Dec;4(12): 1184-7

[10]. Puttick MP, Stein HB, Chan RM, Elwood RK, How AR, Reid GD. Soft tissue tuberculosis : a series of 11 cases. J Rheumatol 1955; 22: $1321-5$

[11]. D'Agta, A.Funto, I Lazzi S and Bonocompagni, G(1997). Video laparoscopic cholecystectomy in a case of biliary lithiasis associated with gall bladder tuberculosis. Minerva Chirurgica, 52(9): 1103-8.

[12]. Nakajo S, Yamamoto M, Urushihira, T Kajitani, T and Tahara, E (1998). Diffuse papillomatosis of the gall bladder complicated with tuberculosis. Acata Pathologica aponica,38(11):1473-80.

[13]. Strelis , A.K. Liadunkin, I,E and Zadorozhnii, A.I(1989). Diagnosis of disorders of bile excretory system in patients with tuberculosis in association with chronic opisthorchiasis. Problemy Tuberkuleza;(1):74-5

[14]. Tanwani R, Sharma D, Chandrakar SK. Tuberculosis of gall bladder without associated gall stone or cystic duct obstruction. Indian J Surg; 2005: 67:45-6.

[15]. Yu R, Lin Y. Gall bladder tuberculosis: case report. Chin Med( Engl) 2002; 115: 1259-61(PubMed) 
[16]. Wafta J, Micheal F. Tuberculose uro-genitale. Prog Urol.2005: 602-603(PubMed)

[17]. SSEN Rabesalama, KL Mandeville, HN Rakato- Ratsimba. Isolated ovarian tuberculosis mimicking ovarian carcinoma: case report and literature review. A fr J. Infect Dis; 2011; 5(1):7-10.

[18]. Harris SH, Khan MA, Khan R, Haque F, Syed A, Ansari MM,.Mammary tuberculosis: Analysis of thirty eight patients. ANZ J Surg:2006;76:234-7(PubMed)

[19]. Tse GM, Poon CS, Ramchandaram K, Ma TK, Pang LM, Law BK,et al. Granulomatous mastitis: A clinicopathological review of 26 cases . Pathology:2004:36: 254-7(PubMed)

[20]. Tiwari M, Shukla HS. Breast tuberculosis: Diagnosis, clinical features and management. Indian J Med.2005;122:103-10(PubMed) 